\title{
La influencia del Programa «La mujer, la paz y la seguridad» en la agenda de Seguridad y Defensa de la Unión Europea*,1
}

\author{
The Influence of the «Women, Peace and Security» Program on the \\ European Union's Security and Defense Agenda
}

\author{
Carolina Jiménez Sánchez \\ Universidad de Málaga \\ carolina@uma.es
}

doi: http://dx.doi.org/10.18543/ced-57-2017pp101-132

Sumario: I. Introducción.-II. La mujer, la paz y la seguridad.III. La implementación del programa «la mujer, la paz y la seguridad» por la Unión Europea. - IV. Planes Nacionales de Acción adoptados por los Estados Miembros de la Unión Europea.-V. El Plan Nacional de Acción de España.-VI. Conclusiones.-VII. Bibliografía.

Resumen: El presente artículo examina el estado del programa «la mujer, la paz y la seguridad» tras diecisiete años desde la adopción de la Resolución 1325, analizando su recepción por la Unión Europea a través de las diferentes herramientas desarrolladas. A su vez, se profundiza en la adopción de Planes Nacionales de Acción por parte de sus Estados Miembros, analizando separadamente el Plan Nacional de Acción español.

Palabras clave: género, mujer, paz y seguridad, Unión Europea (UE), Política Común de Seguridad y Defensa (PCSD), Planes Nacionales de Acción.

Abstract: This article examines the state of the "women, peace and security» program after seventeen years since the adoption of UNSCR 1325, analysing its reception by the European Union through the different tools developed. Besides, the adoption of National Action Plans by EU Member States, specially the Spanish NAP, will be addressed.

Keywords: gender, women, peace and security, European Union (EU), Common Security and Defence Policy (CSDP), National Action Plans.

* Recibido el 12 de enero de 2017 y aceptado el 30 de abril de 2017.

** El presente artículo se ha realizado durante una estancia de investigación en el Centre for Gender and Women's Studies de la Universidad de Lancaster (Reino Unido).

${ }^{1}$ El presente estudio se enmarca dentro del proyecto de investigación: «Las Respuestas del Derecho Internacional y Europeo a los Nuevos Riesgos y Amenazas Contra la Seguridad Humana» (RASEGUR), Plan Nacional de I+D+I (Ref.: DER2015-65906-P) y de la Red de Excelencia sobre «Los actuales desafíos del Derecho Internacional», del Plan Estatal de Investigación Científica y Técnica y de Innovación 2013-2016 (DER15-69273-RED). 


\section{Introducción}

El artículo presenta un estudio pormenorizado de la implementación del programa «la mujer, la paz y la seguridad» en la Unión Europea. Para ello, se han usado diversas metodologías que permiten extraer conclusiones concretas tras más de diecisiete años tras la creación del programa con la Resolución 1325. Entre ellas, el método cuantitativo nos ayudará a determinar cuál ha sido el seguimiento real en la elaboración de los Planes Naciones de Acción por parte de los Estados de la Unión y otros, comparando a su vez los datos de la UE con otros contextos regionales. Además, el estudio va acompañado de un enfoque propositivo y no meramente descriptivo que intenta analizar reflexivamente el resultado de la implementación del enfoque de género realizado en las políticas de la Unión, tomando como punto de referencia su Planteamiento global y sus indicadores.

En primer lugar, se realizará una introducción del programa «la mujer, la paz y la seguridad», haciendo un breve análisis de su recorrido en estos diecisiete años, tomando nota de sus luces y sombras y también reafirmando su enorme valor como punto de inflexión en el reconocimiento de situaciones y Derechos que antes permanecían invisibles. Igualmente, la Resolución 1325 (y siguientes) han puesto el foco de atención en una esfera tradicionalmente androcéntrica, como es la de las negociaciones de paz y el posconflicto. En este sentido, el programa incide en la necesidad de significar y aumentar la participación de las mujeres en los procesos de paz y la reconstrucción posbélica, aspecto que de igual manera será traído a colación.

En segundo lugar, el artículo pretende abordar un estudio de las políticas llevadas a cabo por parte de la UE para implementar el programa «la mujer, la paz y la seguridad», para lo cual ha resultado fundamental su «Planteamiento Global» y el examen de los indicadores. La creación de los indicadores, y su posterior renovación, han permitido la realización de un estudio pormenorizado de los resultados obtenidos hasta el momento en todas y cada uno de las acciones llevadas a cabo relacionadas con la introducción de una perspectiva de género en la seguridad. En este caso, se profundizará especialmente en aquellos indicadores directamente relacionados con procesos de paz de terceros Estados, en los que la Unión Europea está teniendo un rol significativo.

Hecho este análisis, será preciso pasar a estudiar cuál ha sido la recepción del programa por parte de los Estados Miembros de la Unión, para lo que se han recopilado datos de todos los Planes Nacionales de Acción de éstos y de otros Estados y regiones, lo que posibilita un estudio cuantitativo comparativo que además viene reforzado con un análisis en profundidad de algunos de los aspectos más reveladores. Se tomará en cuenta especial- 
mente el Plan Nacional de Acción español, y se reflexionará sobre los retos que plantea, no sólo la adopción, sino la racionalización, diseño y gestión de estos planes.

\section{La mujer, la paz y la seguridad}

La absoluta invisibilidad de los efectos de los conflictos armados en mujeres y niñas, y su discriminación en todas las esferas de la etapa posbélica era patente en la agenda internacional pre-1325. Cuando el Consejo de Seguridad de las Naciones Unidas iniciaba su programa «la mujer, la paz y la seguridad» en el año 2000, con la adopción de la hoy mítica Resolución 1325 , se ponía de manifiesto la necesidad de cambiar el enfoque en los procesos de paz, dando un mayor protagonismo a las mujeres en la resolución de conflictos y dando visibilidad, al fin, a la violencia sufrida por éstas en contextos de violencia sistemática. Diecisiete años después del inicio del programa, y dos años después del Examen de Alto Nivel, las ocho resoluciones que componen el mismo ${ }^{2}$ parecen haber cambiado algo en la concepción del género en la seguridad y en la reconstrucción posbélica ${ }^{3}$. Parece ya una idea generalmente aceptada que en los nuevos contextos bélicos es vital incluir el urgente enfoque de género y llevarlo a las agendas internacionales de seguridad y defensa, siendo una cuestión indispensable en la concepción global de la seguridad.

Adoptado este programa por el Consejo de Seguridad, todos los actores internacionales estaban llamados a implementar medidas que fomentaran la participación de las mujeres en el mantenimiento de la paz y seguridad internacionales, en especial, los Estados y las Organizaciones Internacionales con competencias en seguridad y defensa. Tras diecisiete años, las medidas adoptadas han sido exploradas en el Examen de Alto Nivel y el Estudio Mundial realizado en el año 2015 por ONU Mujeres, después de que el Consejo de Seguridad lo solicitase en su resolución 2122 (2013). Además,

${ }^{2}$ Resolución 1325 (2000) del Consejo de Seguridad de las Naciones Unidas, de 31 de octubre de 2000; Resolución 1820 (2008) del Consejo de Seguridad de las Naciones Unidas, de 19 de junio de 2008; Resolución 1888 (2009) del Consejo de Seguridad de las Naciones Unidas, de 30 de septiembre de 2009; Resolución 1889 (2009) del Consejo de Seguridad de las Naciones Unidas, de 5 de octubre de 2009; Resolución 1960 (2010) del Consejo de Seguridad de las Naciones Unidas, de 16 de diciembre de 2010; Resolución 2106 (2013) del Consejo de Seguridad de las Naciones Unidas, de 24 de junio de 2013; Resolución 2122 (2013) del Consejo de Seguridad de las Naciones Unidas, de 18 de octubre de 2013; Resolución 2242 (2015) del Consejo de Seguridad de las Naciones Unidas, de 13 de octubre de 2015.

3 Kirby, Paul; Shepherd, Laura, «Reintroducing women, peace and security», International Affairs, 92 (2016): 249-254. 
el Secretario General ha expuesto las conclusiones de éste y los progresos del programa en sus informes sobre las mujeres, la paz y la seguridad de septiembre de $2015^{4}$ y en septiembre de $2016^{5}$.

De este balance, al que se ha dado una importancia merecida ${ }^{6}$, sobresale la consagración de la Resolución 1325 como un hito en la historia del feminismo en la Comunidad Internacional. La fuerza transformadora del programa ha hecho evolucionar, ante todo, las «ideas», las concepciones patriarcales sobre la seguridad, la violencia y la paz. Como se expresa en el Estudio Mundial, la resolución era portadora de una idea «sencilla, pero revolucionaria, (que) fue el reconocimiento de que la paz solo puede ser duradera si se incluye plenamente a las mujeres en el proceso, y de que la paz. está unida de manera inseparable a la igualdad entre mujeres y hombres» ${ }^{7}$. Además, su carácter inclusivo de todas las esferas relacionadas con la paz y la seguridad ha otorgado un lugar especial a los mecanismos de protección de los Derechos Humanos y a la promoción de la eliminación del techo de cristal en las altas esferas políticas y militares. En su esencia se encuentra, cómo no, el concepto de mainstreaming ${ }^{8}$, que trata no de asignar un espacio al género sino que canalizar la perspectiva de género en todos y cada uno de los elementos de la paz, la seguridad y la violencia.

Tanto es así que dicho Estudio Mundial recomienda a los Estados eliminar las reservas que puedan tener a la CEDAW, e informar sobre los progresos en el programa «la mujer, la paz y seguridad» en el EPU ${ }^{9}$. Es importante en la medida en que la CEDAW ha sido objeto de reservas por parte

${ }^{4}$ Informe del Secretario General sobre las mujeres, la paz y la seguridad, S/2015/716, de 16 de septiembre de 2015 .

5 Informe del Secretario General sobre las mujeres, la paz y la seguridad, S/2016/822, de 29 de septiembre de 2016.

${ }^{6}$ Huguuet, Montserrat; Folguera, Pilar, «Seguridad Internacional y Mujeres en los retos de Naciones Unidas para el mantenimiento de la paz», Revista de Historia Contemporánea AYER, n. ${ }^{\circ} 101$ (2016): 285-297.

7 Prevenir los conflictos, transformar la justicia, garantizar la paz, Estudio Mundial sobre la aplicación de la Resolución 1325 del Consejo de Seguridad de las Naciones Unidas, ONU Mujeres (2015): 28. El Estudio mundial puede consultarse en: http://wps.unwomen.org/en

${ }^{8}$ El mainstreaming de género fue asumido como concepto en la Plataforma de Acción de Beijing. Según el Grupo de Expertos del Consejo de Europa se puede definir como «la organización (la reorganización), la mejora, el desarrollo y la evaluación de los procesos políticos, de modo que una perspectiva de igualdad de género se incorpore en todas las políticas, a todos los niveles y en todas las etapas, por los actores normalmente involucrados en la adopción de medidas políticas», (Mainstreaming de género. Marco conceptual, metodología y presentación de «buenas prácticas». Informe final de las actividades del Grupo de especialistas en mainstreaming (EG-S-MS), Consejo de Europa (versión español e inglés), Instituto de la Mujer, Ministerio de Trabajo y Asuntos Sociales, Serie documentos, número 28, Madrid, 1999, pp. 26).

${ }^{9}$ Examen Periódico Universal realizado por el Consejo de Derechos Humanos. 
de la mayoría de Estados que lo han ratificado, ya sea por motivaciones religiosas (16 Estados), político-institucionales (17 Estados), económicas (5) o por los mecanismos de control (40 Estados $)^{10}$. La inclusión de una atenta mirada a la protección de Derechos Humanos es fundamental teniendo en cuenta que la discriminación y Violencia Basada en el Género (VBG) que ocurre en el conflicto es sólo una exacerbación de los roles de género existentes en etapas previas. Por ello, la implementación de medidas del programa no sólo va dirigida a los Estados que se encuentren en conflictos armados o procesos de justicia transicional, sino a todos los sujetos de la Comunidad Internacional. Es decir, que el programa «la mujer, la paz y la seguridad» va mucho más allá de los contextos de conflicto y posconflicto. Su intención última debe ser penetrar en todas las etapas de la discriminación, que empiezan y acaban con los Derechos Humanos. Como declara el Secretario General en su informe de 2015 «la prevención de conflictos con una perspectiva de género debe partir de una comprensión de la inseguridad, las desigualdades y las violaciones de los derechos humanos que afectan a la vida de las mujeres y las niñas antes, durante y después de los conflictos» ${ }^{11}$.

En octubre de 2015 España, en su calidad de miembro no permanente del Consejo de Seguridad, presidía la sesión del mismo en la que se llevaría a cabo el debate abierto y el Examen de Alto Nivel sobre las mujeres, la paz y la seguridad, y en la que sería adoptaba la Resolución 2242 (2015) que, hasta el momento es la última del programa. La propuesta de resolución, elaborada por España y Reino Unido y que fue aprobada por unanimidad, supone una recapitulación del trabajo realizado y los retos pendientes tras 15 años desde la aprobación de la 1325. En ella se insta a los Estados a re-evaluar sus estrategias y a la asignación certera de recursos a actividades del programa, a la luz de las carencias detectadas en el Estudio Mundial publicado en el mismo año. Esta idea estaba ya inserta en el citado Informe del Secretario General del año 2015, en el que alienta «a todos los agentes que participan en los procesos de paz a contraer compromisos cuantificables, con plazos concretos, de asegurar la participación directa y productiva de las mujeres en todas las fases del proceso» ${ }^{12}$. Como una herramienta más del control de esos progresos, en dicha Resolución se propuso también

10 Magdalena Martín Martínez y Carolina Jiménez Sánchez, «La protección internacional de los Derechos Humanos de las mujeres: una visión desde la multiculturalidad y la perspectiva de género», en Diversidad Cultural, Género y Derecho, ed. por Patricia Laurenzo Copello y Rafael Durán Muñoz (Tirant lo Blanch, 2014): 269-305.

11 Informe del Secretario General sobre las mujeres, la paz y la seguridad, S/2015/716, de 16 de septiembre de 2015, párr. 67.

12 Ibidem., párr. 154. 
la creación de un Grupo Oficioso de Expertos del Consejo de Seguridad sobre la mujer, la paz y la seguridad, que actualmente preside España junto a Reino Unido ${ }^{13}$.

La exigencia del Secretario General en cuanto a la adquisición de «compromisos cuantificables» reside en los múltiples desafíos que todavía hoy socavan la efectividad de la Resolución 1325 y siguientes ${ }^{14}$. De hecho, la Resolución 2242 se dedica también evidenciar los puntos débiles del programa, entre ellos: los episodios de violencia sexual que se siguen sucediendo por parte de los agentes de las $\mathrm{OMP}^{15}$ (situación extremadamente grave cuya responsabilidad se encuentra fundamentalmente en la propia Organización de las Naciones Unidas, así como en los Estados Miembros que envían personal), la VGB que se sigue produciendo en los conflictos, la inactividad de los Estados frente a la infra-representación de las mujeres en todas las esferas de toma de decisiones o, por vez primera, las implicaciones de género existentes en el extremismo violento y la lucha antiterrorista.

El Examen de Alto Nivel ha logrado dar un impulso al programa, que dos años después sigue recibiendo propuestas e iniciativas por parte de algunos Estados. En septiembre de 2016 también España lanzaba la red de coordinadores nacionales sobre la Resolución 1325, cuya primera reunión tendrá lugar en España en 2017. En aspectos más concretos, Canadá propuso la creación de una red de asesores de género militares y de policía. El Grupo Oficioso ha mantenido por el momento diversas reuniones en las que examina la situación del programa «la mujer, la paz y la seguridad» en situaciones específicas, como la de Afganistán, Irak ${ }^{16}$, Malí y República Centroafricana ${ }^{17}$, dando cuenta de las necesidades y los retos que enfrentan las mujeres y niñas en dichos escenarios. Este es un camino óptimo que incide, no ya en el establecimiento de un marco general de ac-

13 Actas de la 7793. a sesión del Consejo de Seguridad de las Naciones Unidas, de 25 de octubre de 2016, S/PV.7793, p. 7. En palabras del representante español: «un mecanismo que pone a disposición de los miembros del Consejo de Seguridad información concreta de lo que necesitamos obtener en materia de liderazgo en las Naciones Unidas. Es también un mecanismo al servicio de las misiones de paz, para apoyar sus esfuerzos por integrar la perspectiva de género de manera generalizada en las operaciones de mantenimiento de la paz».

${ }^{14}$ María Solanas, «Mujeres, paz y seguridad: lejos de las aspiraciones de la Resolución 1325», ARI 44/2015, Real Instituto Elcano, 15 de septiembre de 2015.

${ }^{15}$ La Resolución 2242 (2015) del Consejo de Seguridad de las Naciones Unidas, de 13 de octubre de 2015 expresa en su punto 9 «profunda preocupación por las persistentes denuncias de actos de explotación cometidos por el personal de mantenimiento de la paz de las Naciones Unidas y los fuerzas ajenas a la Organización».

$16 \mathrm{~S} / 2016 / 683$.

$17 \mathrm{~S} / 2016 / 672$. 
tuación, sino en la ejecución del mencionado mainstreaming o enfoque de género, aplicándolos a situaciones concretas sobre el terreno y detectando los desafíos, que no son del mismo tipo en todos los contextos. En el caso de Afganistán, éstos tienen que ver con «the need to ensure gender-sensitive electoral processes and the need for safe work environments for women in the security sector, in order to improve participation and representation of women in public spheres» ${ }^{18}$, mientras que en Malí existen enormes problemas con la MGF (que ronda el 90\%) y los matrimonios forzados ${ }^{19}$, así como con «messages against gender equality in social media (...) being used to radicalize and recruit young people in the north». El Grupo Oficioso realiza en cada una de las situaciones unas recomendaciones sobre cómo implementar el programa en relación a los desafíos concretos de cada territorio.

En esta nueva etapa del programa «la mujer, la paz y la seguridad», hay que confirmar la suficiencia del marco normativo general, desplazando el programa a una nueva fase en la que se deben asumir compromisos concretos y focalizar los esfuerzos de toda la Comunidad Internacional en las mayores amenazas a la seguridad de las mujeres y niñas que hay actualmente en el planeta, en línea con el trabajo del Grupo Oficioso. Como apunta el Secretario General en su informe sobre las mujeres, la paz y la seguridad de 2016 la estrategia a seguir en la siguiente etapa del programa es la de «rendición de cuentas por resultados» 20 .

Desde un análisis en perspectiva, el programa ha tenido un efecto a medio plazo, como esbozaba arriba, de «ideas», de cambio en las concepciones tradicionales sobre la paz, la violencia, la seguridad y la toma de decisiones. Todo avance ideológico conlleva, no obstante, un tiempo de recepción en los agentes tradicionales anclados a esquemas patriarcales por más de veinte siglos. Tras diecisiete años de «la mujer, la paz y la seguridad» nadie duda de que han cambiado los equilibrios en las representaciones sobre la seguridad y la paz, como también han cambiado algunos gráficos que ahora muestran tendencias hacia la igualdad cuando antes la norma era la ausencia de gráficos sobre tema alguno de género. Y este es, además, un logro global.

Todo éxito del programa pasa necesariamente por su internacionalización y por aunar esfuerzos entre la sociedad civil, los Estados y las Organizaciones Internacionales para así dotar de las herramientas adecuadas para los desafíos permanentes (generales y específicos) que, hoy

\footnotetext{
$18 \mathrm{~S} / 2016 / 673$.

$19 \mathrm{~S} / 2016 / 682$.

${ }^{20}$ Informe del Secretario General sobre las mujeres, la paz y la seguridad, S/2016/822, de 29 de septiembre de 2016, párr. 110.
} 
se encuentran más que nunca identificados, analizados y cuantificados. Tenemos, pues, el marco normativo suficiente para avanzar un paso más hacia aquello tan falsamente utópico de la igualdad real y efectiva, que significa mucho más que la igualdad por sí sola. En este sentido, el Secretario General en su informe sobre las mujeres, la paz y la seguridad de 2016, afirma que los Estados «siguen siendo los que están en mejor disposición de influir en la aplicación de la agenda relativa a las mujeres, la paz y la seguridad, en su calidad de Miembros de las Naciones Unidas y de organizaciones regionales, partes en los conflictos, donantes, países que aportan contingentes y fuerzas de policía a las operaciones de mantenimiento de la paz e interlocutores políticos en un conflicto o región concretos. Tienen la responsabilidad primordial de garantizar los compromisos mundiales asumidos en el marco de la agenda se integren en las políticas y legislaciones nacionales» ${ }^{21}$. Así, es una cuestión fundamental y poco examinada cuál es la recepción que ha tenido el programa en la Unión Europea, asunto de máxima importancia por ser ésta una organización regional de integración y con competencias en seguridad y defensa, que debe velar también por ser modelo para sus Estados Miembros en el seguimiento de las orientaciones de las Naciones Unidas.

\section{La implementación del programa «la mujer, la paz y la seguridad» por la Unión Europea}

Constatada la existencia de un mandato global para incluir las propuestas del programa «la mujer, la paz y la seguridad» (en adelante, MPS) así como la necesidad de evaluar las acciones realizadas hasta el momento, resulta fundamental analizar cuál ha sido el camino seguido por la Unión Europa a lo largo de la corta vida del programa, examinando si existe también cierta consonancia con las tendencias de sus Estados Miembros y otros contextos regionales. El interés de dicha propuesta es, si cabe, mayor, por tratarse de una Organización con fuertes implicaciones en cuestiones de seguridad y defensa, que actualmente se enfrenta a nuevos desafíos en dicho campo. Además tiene en su haber, por ejemplo, la capacidad de desplegar misiones y operaciones que se desplazan a territorios en conflicto, así como cierta experiencia en mediar en conflictos internacionales.

${ }^{21}$ Informe del Secretario General sobre las mujeres, la paz y la seguridad, S/2016/822, de 29 de septiembre de 2016, párr. 68. 
Sus significativos atributos en la acción exterior juegan un papel crucial en el cómputo global de la paz y la seguridad, por lo que sería enormemente dañino que la Unión Europea no hubiera realizado acciones positivas a favor de integrar la perspectiva de género en su agenda de seguridad. En efecto, esto fue así durante los primeros años de vida de la Resolución 1325, pero se fue tomando con mayor seriedad a raíz de la Resolución 1820, que, en 2008, instaba a «los órganos regionales y subregionales apropiados en particular a que examinen la posibilidad de elaborar y aplicar políticas y realizar actividades y tareas de promoción en beneficio de las mujeres y las niñas afectadas por la violencia sexual en los conflictos armados» 22 .

En el mismo año, el Consejo de la Unión Europea aprobaba un importante documento con el objeto de integrar una perspectiva de género en todas las áreas conectadas con los conflictos armados, el posconflicto y la reconstrucción, es el «Planteamiento global de la UE sobre la aplicación de las Resoluciones 1325 y 1820 del Consejo de Seguridad de las Naciones Unidas sobre la mujer, la paz y la seguridad» ${ }^{23}$, que ha sido visto como un plan de acción regional. En el mismo, se reconocía, además, la conexión entre la seguridad, la paz y el desarrollo con la igualdad de género ${ }^{24}$ y se trataba de crear un marco común de actuación y de evaluación de las políticas y acciones relacionadas con la paz y la seguridad, así como con la cooperación con terceros Estados. Como medidas específicas, se pone especial énfasis en el apoyo político a las resoluciones del programa MPS, en ofrecer una formación sensible al género en todos los componentes conectados con la paz y la seguridad, como las relacionadas con la Reforma del Sector de la Seguridad (SSR) o cooperar con otros actores internacionales, tanto Organizaciones Internacionales como Estados, en particular aquellos inmersos en procesos de paz. Igualmente, se establece la necesidad de evaluación y monitoreo de las acciones que lleve a cabo la Unión, para lo que se crea la «Women, Peace and Security Task Force ${ }^{25}$, con el objetivo de fomentar la coordinación y promover un correcto acercamiento a las cuestiones relacionadas con el género, de conformidad con las líneas marcadas en el Planteamiento global.

22 Punto 14 de la Resolución 1820 (2008) del Consejo de Seguridad de las Naciones Unidas, de 19 de junio de 2008.

23 Planteamiento global de la UE sobre la aplicación de las Resoluciones 1325 y 1820 del Consejo de Seguridad de las Naciones Unidas sobre la mujer, la paz y la seguridad, 15671/08, Bruselas, 1 de diciembre de 2008.

24 Ibidem., párr. 15.

25 Ibidem., párr. 28. 
En el documento se establecen unas orientaciones comunes para implementar las recomendaciones del programa «la mujer, la paz y la seguridad», basadas en el llamado triple enfoque según el cual es necesario que la Unión Europea:

1) Integre las líneas marcadas por la Resolución 1325 (2000) y 1820 (2008) en su diálogo político con los Estados particularmente afectados por el conflicto o el posconflicto.

2) Realice un gender mainstreaming en todas sus políticas y activida$\operatorname{des}^{26}$.

3) Apoye acciones específicas y estratégicas para el empoderamiento de las mujeres ${ }^{27}$.

En esta línea, la UE incorporaría las directrices de la resolución 1325 a su marco normativo en tres áreas prioritarias: la protección de las mujeres víctimas de los conflictos armados, la participación activa de las mujeres en los procesos de paz y el papel de éstas en la prevención de $\operatorname{conflictos}^{28}$. Es evidente que las estrategias encaminadas a homogeneizar prácticas de los Estados en cualquiera de estos tres terrenos deben venir precedidas del ejemplo de la propia Unión, sobre todo respecto a la participación activa de mujeres tanto en la prevención de conflictos como en su participación en los procesos de paz.

En el año 2010 el Consejo reforzaba su actividad sobre el enfoque común y el seguimiento de las orientaciones diseñadas en 2008, a través de la «Women, Peace and Security Task Force», a la que se le encargaba la elaboración de unos indicadores en línea con el Planteamiento global, sobre el progreso de la protección y el empoderamiento de las mujeres en contextos de conflicto y posconflicto ${ }^{29}$. Meses antes el Secretario General de las Naciones Unidas ya había establecido 26 indicadores que incluían las líneas

${ }^{26}$ Sobre los orígenes de este particular Vid., Emanuela Lombardo, «El Mainstreaming de género en la Unión Europea», Aequalitas. Revista jurídica de igualdad de oportunidades entre mujeres y hombres, vol. 10-15, mayo-diciembre (2003): 6-11.

27 Planteamiento global..., op. cit., párr. 18.

${ }^{28}$ Barbé, Esther, «Supporting Practices Inspiring by Solidarist Ideas: The EU and the UNSC Open Debates on Women, Peace and Security», en EU Policy Responses to a Shifting Multilateral System (Barbé, Esther, et al), Palgrave Macmillan (2016): 135-156.

29 Éstos debían estar basados en los que se habían diseñado un año antes para vigilar el respeto de los principios de la Plataforma de Acción de Beijing. El párrafo 43 del Planteamiento global (original en inglés) establecía: «On the basis of the four indicators elaborated under the French Presidency for the follow-up of the Beijing Platform for Action area of concern «Women and armed conflicts», the "Women, Peace and Security Task Force" will develop further indicators for progress regarding the protection and empowerment of women in conflict settings and in post conflict situations». 
principales del programa ${ }^{30}$, respondiendo al requerimiento del Consejo de Seguridad en la Resolución 1820 (2008) ${ }^{31}$. La Unión, por su parte, estableció 17 indicadores ${ }^{32}$ específicos que serían complementados por los de las Naciones Unidas para cuestiones que sean de orden global ${ }^{33}$.

Divididos en cuatro áreas, los 17 indicadores se referían a la prevención de conflictos, la participación de las mujeres en los procesos de paz, la protección frente a la violencia ejercida en dichos contextos así como la vigilancia de los Derechos Humanos, la ayuda humanitaria y la recuperación. El establecimiento de unos indicadores demuestra un mayor compromiso con la aplicación del programa MPS. Este ya no constará sólo de recomendaciones a los Estados, sino que se concreta en situaciones cuantificables que permiten observar el progreso o no de las herramientas que han usado los Estados y las Organizaciones para poner en práctica dichas recomendaciones. Es, por tanto, un camino óptimo para comprometerse con resultados concretos que lleven a las sociedades hacia la igualdad real y efectiva. Del mimo modo, la realización de un auténtico enfoque integral de género hace necesaria la observación de cada variable por separado para hacerlo posteriormente de manera global, pues la integración de una perspectiva de género conlleva un ejercicio complejo de reformulación de políticas, protocolos, prácticas de las más diversa índole que ha de hacerse desde el interior de cada órgano y no desde un «órgano rector del género» que contemple directrices generales y no mandatos específicos sobre los elementos de transformación. Por otro lado, es cierto que el diseño de los indicadores responde a una etapa previa de verificación del estado de la cuestión, sin embargo, no se han dotado de metas a alcanzar como en otros compromisos cuantificables similares, basten como ejemplo los Objetivos de desarrollo del Milenio o los Objetivos de Desarrollo Sostenible impulsados por las Naciones Unidas. La asunción de metas en relación con los indicadores en el programa MPS sigue siendo un asunto pendiente, que debería desarrollarse en etapas posteriores.

Los 17 indicadores establecidos por la «Women, Peace and Security Task Force» se muestran de manera abreviada en la siguiente tabla:

30 UN Strategic Results Framework on Women, Peace and Security: 2011-2020, accessible en http://www.un.org/womenwatch/ianwge/taskforces/wps/Strategic_Framework_20112020.pdf.

31 Resolución 1820 (2008) del Consejo de Seguridad de las Naciones Unidas, de 19 de junio de 2008, párr. 17.

32 Indicators for the Comprehensive Approach to the EU implementation of the United Nations Security Council Resolution 1325 and 1820 on women, peace and security, 11948/10, Bruselas, 14 de julio de 2010.

33 Ibidem., p. 7. 
$1 \quad$ N. ${ }^{\circ}$ de países socios de la UE a los que se apoya en algún tipo de acción sobre MPS

2 Herramientas e instrumentos de financiación en apoyo a MPS en países socios

\begin{tabular}{|c|c|}
\hline 3 & N. ${ }^{\circ}$ diálogos regionales sobre MPS \\
\hline 4 & $\begin{array}{l}\text { N. de países socios en los que las acciones sobre MPS se coordinan por EM } \\
\text { y/o otros }\end{array}$ \\
\hline 5 & $\begin{array}{l}\text { N. proyectos de cooperación en terceros Estados con objetivos de igualdad } \\
\text { de género }\end{array}$ \\
\hline 6 & N. ${ }^{o}$ Planes Nacionales de Acción y otros documentos estratégicos en los EM \\
\hline 7 & N. o de iniciativas el colaboración con la ONU y otras OOII sobre MPS \\
\hline 8 & N. ${ }^{o}$ y porcentaje de mujeres negociadoras y mediadoras apoyadas por la UE \\
\hline 9 & $\begin{array}{l}\text { Actividades de la UE en apoyo de la participación de las mujeres en los } \\
\text { procesos de paz }\end{array}$ \\
\hline 10 & $\begin{array}{l}\text { N. o y tipo de reuniones (con mujeres) de la UE, las delegaciones de los EM y } \\
\text { las misiones PCSD sobre MPS }\end{array}$ \\
\hline 11 & $\begin{array}{l}\text { Proporción por sexo de jefes de misiones diplomáticas, delegaciones y mi- } \\
\text { siones PCSD. Proporción por sexos personal enviado a las OMP de la ONU. }\end{array}$ \\
\hline 12 & $\begin{array}{l}\text { Proporción por sexos de personal diplomático, civil y militar con formación } \\
\text { en igualdad de género }\end{array}$ \\
\hline 13 & $\begin{array}{l}\text { N. }{ }^{\circ} \text { y porcentaje de misiones PCSD que incluyan la igualdad y los principios } \\
\text { de MPS }\end{array}$ \\
\hline 14 & N. y porcentaje de misiones PCSD con asesores de género \\
\hline 15 & $\begin{array}{l}\text { N. casos de abuso y explotación sexual en las misiones PCSD y medias to- } \\
\text { madas }\end{array}$ \\
\hline 16 & $\begin{array}{l}\text { Porcentaje de informes de actividad de los Representantes especiales de la UE } \\
\text { que incluyan información sobre MPS }\end{array}$ \\
\hline 17 & $\begin{array}{l}\text { Proporción, país de origen y sexo de los solicitantes de asilo que han obtenido } \\
\text { protección internacional }\end{array}$ \\
\hline
\end{tabular}

Buena parte de los indicadores se basan en la cooperación internacional, ya sea con terceros Estados o con otras Organizaciones Internacionales. Seña de normalidad teniendo en cuenta que la UE es, en esencia, un actor de coopera en procesos de paz de terceros y que, además, tiene un papel fundamen- 
tal en la prevención de los conflictos. Igualmente, hay que tener en cuenta que se trata de un enfoque cuantitativo del programa MPS, aunque se incluyen algunos elementos de examen cualitativo que atienden al contenido y no tanto al número, como el número 2 o el 9 . No obstante, es relevante conocer las tendencias y los «números» de los objetivos que se alcancen, pues es un reto primario para la consecución de la igualdad. La ausencia de datos en diferentes áreas es un síntoma muy claro de la necesidad de analizar de manera profunda la situación y evolución de los objetivos propuestos.

En mayo de 2011 se publica un primer informe sobre los progresos alcanzados en cada uno de los indicadores ${ }^{34}$, que ponía de manifiesto cuáles eran por el momento los puntos débiles de la UE, a saber: la participación de las mujeres en los procesos de paz, la incorrecta interpretación de los Planes Nacionales de Acción de los EM o el número de mujeres representantes de las delegaciones de éstos hacían identificables los retos concretos en los que poner un mayor énfasis. En el segundo informe ${ }^{35}$ adoptado en 2014, la información recibida por parte de los EM es menor (pasa de ser de un $89 \%$ en el primer informe a un 59\% en el segundo), con lo cual se percibe un desvalor de las cuestiones de MPS en las agendas de los EM. De esta manera, como limitación, los datos no pondrán de manifiesto la realidad de los $28 \mathrm{EM}$, sino sólo de la de aquellos que han suministrado información, lo que ya por sí solo es muestra de interés en el programa.

Esta falta de información se hace patente doblemente en el indicador n. ${ }^{\circ} 8$. El primer informe se limita a poner de manifiesto que no existen datos para cuantificar número alguno de mujeres negociadoras y, en el segundo informe se propone la elaboración de un proyecto que tenga como objetivo la elaboración de estos datos, por lo que por el momento no se pueden establecer conclusiones salvo la de significar que la ausencia de datos es siempre una muestra de la falta de importancia dada a la cuestión. Sin embargo, a la luz del Estudio Mundial podemos conocer datos relevantes en la materia (no en términos cuantitativos pero sí cualitativos). En el acuerdo histórico por el que Irán daba marcha atrás en su programa nuclear ${ }^{36}$ fue gestado en buena medida por Catherine Ashton, Jefa de la diplomacia europea

${ }^{34}$ Report on the EU Indicators for the Comprehensive Approach to the EU implementation of the UNSCRs 1325 and 1820 on women, peace and security (2011-9990/11)

35 Second Report on the EU-indicators for the Comprehensive Approach to the EU Implementation of the UN Security Council Resolutions 1325 \& 1820 on Women, Peace and Security (2014-6219/14)

36 Plan de Acción Integral Conjunto (PAIC) concertado el 14 de julio de 2015 (S/2015/544), recogido en la Resolución 2231 (2015) del Consejo de Seguridad de las Naciones Unidas, de 20 de julio de 2015. 
hasta finales de 2014. En las arduas negociaciones de 2015 la Alta Representante Federica Mogherini, junto con la diplomática alemana Helga Schmid y la estadounidense Wendy Sherman, ejercieron labores de mediación cruciales para que el acuerdo tuviera lugar ${ }^{37}$. En el caso del proceso de paz colombiano, que en un primer momento no contaba con mujeres negociadoras, el cambio de óptica fue fomentado desde los foros internacionales feministas y aplaudido por el Parlamento Europeo en su resolución en apoyo al proceso de paz, en la que elogia «el establecimiento de una Subcomisión de Género que garantice la integración de la perspectiva de género en las negociaciones y la participación de las víctimas de violencia sexual y de las organizaciones defensoras de los derechos de las mujeres en los diálogos de paz no tienen precedentes y deberían ser una inspiración para otros procesos de paz en todo el mundo» ${ }^{38}$. La relación positiva entre la inclusión de mujeres en los procesos de negociación y mediación y los acuerdos alcanzados ha sido puesta de manifiesto por diversos estudios empíricos en los que se constata dicha sinergia objetiva ${ }^{39}$.

El peso de la Unión Europea como sujeto mediador es crucial, sin embargo no hay que desmerecer la labor de cooperación reforzada que realizan algunos EM en este sentido. Por ejemplo, en el año 2012 España sacaba adelante en colaboración con Marruecos una iniciativa para promover el papel de las mujeres como mediadoras en el Mediterráneo, lo que está permitiendo la formación de éstas y su puesta a disposición a las Naciones Unidas y de las organizaciones regionales ${ }^{40}$. No obstante, la iniciativa pierde fuelle con acciones como la expulsión de la Vicepresidenta del Parlamento de la Unión Africana, la saharaui Suilma Beiruk, durante la cumbre de Naciones Unidas sobre el cambio climático en Marrakech en noviembre de $2016^{41}$.

La influencia en los procesos de paz también se mide mediante el indicador $\mathrm{n} .^{\circ} 11$, respecto al que sí se ofrecen datos claros, y la tendencia existente es positiva. En el primer informe sólo se detecta una mujer como jefa de un Delegación en países de frágil estabilidad y 10 jefas en las Delegacio-

37 Prevenir los conflictos..., op. cit., p. 40.

38 Resolución del Parlamento Europeo en apoyo al proceso de paz en Colombia, 2015/3033(RSP), de 19 de enero de 2016.

39 Paffenholz et al., «Making Women Count: Assessing Women's Inclusion and Influence on the Quality and Sustainability of Peace Negotiations and Implementation», Geneva: Inclusive Peace and Transition Initiative (The Graduate Institute of International and Development Studies and UN Women, April 2016): 61.

40 Actas de la 7793. a sesión del Consejo de Seguridad de las Naciones Unidas, de 25 de octubre de 2016, S/PV.7793, p. 71.

${ }^{41}$ Como se referencia en la edición digital de La Vanguardia del 7 de noviembre de 2016. Accesible en: http://www.lavanguardia.com/internacional/20161107/411665076688/ marruecos-expulsa-del-pais-a-la-vicepresidenta-del-parlamento-africano.html. 
nes de todos los Estados, siendo un 18\% del total. En el segundo informe, tres años después el porcentaje ha ascendido hasta el $21 \%$, habiendo un total de 24 mujeres jefas de Delegación. Sin embargo no se especifica cuántas corresponden a Estados frágiles o en conflicto. En el personal de las misiones y operaciones se aprecia el persistente techo de cristal, y también una desproporción de la participación de las mujeres en las mismas, que puede ir desde el 7\% en la Misión BAM Rafah hasta el 38\% en la Misión EUMM Georgia.

Una de las prioridades de la integración de las políticas de MPS en la UE debería identificarse con el indicador $n .^{\circ} 13$, pues como actor esencial en procesos de reconstrucción en terceros Estados, las misiones de la UE tienen la capacidad de poner en marcha acciones y políticas vitales para los territorios en conflicto o posconflicto. Sin embargo, este indicador revela importantes deficiencias en el primer informe que descubren la no inclusión plena de los objetivos MPS en el interior de sus misiones. En el primer período examinado sólo habían incorporado estos objetivos a los mandatos de 2 de las 13 misiones y operaciones desplegadas. En el segundo informe ascendían hasta la mitad de los mandatos (7 de 14). Sin duda, es un aumento considerable pero sigue sin ser plausible que la mitad de las misiones y operaciones de la UE continúen sin incluir estos objetivos en su mandato. No obstante, todas ellas comprenden, según ambos informes, referencias claras a las cuestiones de género, pese a que sólo la mitad lo incluya en el mandato. Es clara muestra también de la importancia con que se toma la cuestión la manera de proceder a incluir «la perspectiva de género» en las políticas. Definitivamente no es igual incluirla en protocolos, reglamentos o documentos orientativos que hacerlo en el mandato de una misión u operación, que es la columna vertebral de la misma. Además, el enfoque de género conlleva asociarlo de manera continua y permanente a todos los documentos operacionales de la misión, lo que fomentaría la mejor adaptación del personal a un método de trabajo con enfoque de género, lo que actualmente no se refleja en el día a día de algunas misiones, como se ha puesto de manifiesto al respecto de la Misión EUMM Georgia ${ }^{42}$ pese a ser, no obstante, la que mayor porcentaje de mujeres tiene.

La existencia de asesores de género en el interior de las misiones y operaciones suponen un avance en la calidad e integración de la perspectiva de género, tan necesaria en este ámbito ${ }^{43}$. Su tarea consiste en recomendar medidas para mejorar el enfoque de género en las misiones y operaciones. En este sentido, la información proporcionada sobre el indicador $n .^{\circ} 12$ indica

${ }^{42}$ Louise Olsson et. al., Gender, Peace and Security in the European Union's Field Missions, Folke Bernardotte Academy, Stockholm (2014): 32.

43 Álvarez, Alejandro José, «Mujer, paz y seguridad y los nuevos desafíos a la seguridad global», VI Congreso de Relaciones Internacionales, La Plata (2016). 
un descenso en la inclusión de asesores de género en el segundo período, existiendo un asesor de género en el $75 \%$ de las operaciones y en el $60 \%$ de las misiones, mientras que en el primer informe existía un $90 \%$ del total de operaciones y misiones con un experto en género. El adiestramiento sensible al género en las misiones ya se venía contemplando desde el año 2008 cuando se adoptó la tercera revisión del documento «Implementation of UNSCR 1325 in the context of ESDP» ${ }^{44}$. En este se establece que el Comandante debe recibir adiestramiento sobre la implementación de la Resolución 1325, y el resto del personal debe recibir formación sensible al género, en especial sobre la violencia sexual. Se establecía cierta prioridad de la prevención de la violencia sexual, en lo que se ha denominado como política de tolerancia cero ${ }^{45}$.

En este sentido, respecto al indicador n..$^{\circ} 15$, en el período cubierto por el primer informe se identificaron 6 casos de abuso y explotación sexual, provenientes de 4 misiones. En el segundo informe ascienden a 13, provenientes de sólo de 2 misiones. Los datos son reveladores, pues es posible que el aumento de denuncias sea consecuencia no de una mayor violencia, sino de un mayor índice de denuncias consecuencia de la visibilización del problema. En el segundo informe se afirma que todos los casos fueron investigados y tuvieron como consecuencias 5 repatriaciones y una sanción disciplinaria.

La política de repatriaciones cuando se constate la existencia de conductas de violencia sexual sigue la reciente línea establecida en la Resolución 2272 (2016) del Consejo de Seguridad ${ }^{46}$, en la que se consagra esta nueva sanción recomendada por el Secretario General, cuando existan pruebas de dicha violencia. Además, en el caso de que el Estado responsable no tome medidas disciplinarias la Resolución propone sustituir a todas las fuerzas militares y/o policiales de dicho Estado, necesidad que, como ya adelanté en publicaciones anteriores ${ }^{47}$, era urgente para otorgar coherencia al programa MPS y su obligada y estrecha relación con las operaciones de paz y la pretendida tolerancia cero. De esta manera, si se verifica que un EM no

${ }^{44}$ Implementation of UNSCR 1325 in the context of ESDP, Doc. 15782/3/08 REV. 3 En 2005 se habían ya adoptado mencionado en las conclusiones del Consejo en «Council conclusions on ESDP, GAERC, 23 de mayo de $2005 \mathrm{y}$ en «Implementation of UNSCR 1325 in the context of ESDP, Doc., 11932/2/05 REV 2.

45 Cristina Amich Elías, «Capacitación, monitoreo e indicadores», en Formación y adiestramiento sensible al género del personal cívico-militar y el empoderamiento de la mujer, Susana de Tomás Morales (Dir.), Dyckinson y Ministerio de Asuntos Exteriores y Cooperación (2015): 241.

46 Resolución 2272 (2016) del Consejo de Seguridad de las Naciones Unidas, de 11 de marzo de 2016.

47 En este sentido, Vid., Carolina Jiménez Sánchez, Las mujeres en los conflictos armados: conflicto, proceso de paz y posconflicto (SPYDUM, Málaga, 2016): 276. 
cumple con los mínimos contemplados en el programa MPS, el Secretario General recomendará que éste no envíe de personal para las OMP. Por ello, es muy necesario que la UE profundice en el análisis de este indicador y, además, se realice un esfuerzo por otorgar coherencia desde el marco regional a las directrices marcadas por el Consejo de Seguridad, exigiendo responsabilidades a nivel interno en las conductas criminales detectadas también en las misiones y operaciones de la UE.

Otro de los retos de mayor complejidad que debe afrontar la UE en el presente decenio es el relativo a la crisis humanitaria de refugiados. Como hemos podido observar, la falta de asunción de compromisos de manera apacible por parte de algunos EM hace aún más compleja la gestión de estos flujos migratorios. A ello hay que sumar lo poco o nada que se ha debatido la necesidad de integrar perspectiva de género, ya sea a nivel regional o estatal, en cuanto a las solicitudes de asilo y la situación de las mujeres y niñas refugiadas, que se encuentran además en el punto de mira de numerosas organizaciones de trata de personas con fines de explotación y esclavitud sexual. En el indicador $\mathrm{n} .^{\circ} 17$, el primer informe establecía la inexistencia de datos desagregados por sexos respecto a los solicitantes de asilo, mientras que en el segundo 12 EM ofrecieron estos datos, cuyo resultado medio es que el $36 \%$ de los solicitantes de asilo son mujeres. De éstas, un $13 \%$ consiguió asilo y otro idéntico $13 \%$ consiguió protección subsidiaria. Es muy necesario que los datos hubieran mostrado también las causas por las que las mujeres solicitan asilo, pues el hecho de que pueda tratarse de persecución de género debería transformar el enfoque y las políticas de protección así como el examen de su solicitud y las conexiones con el programa MPS. Igualmente, hay que destacar que la ausencia de datos sigue existiendo en $16 \mathrm{EM}$, entre los que se encuentra España, Italia o Hungría. Siendo, estos dos últimos, Estados clave en la recepción de refugiados en los últimos años.

La mayor aportación del segundo informe de revisión de los indicadores es la petición de renovación de los mismos, que cuatro años después de ser establecidos y tras los dos exámenes sobre los progresos se consideran desfasados o se han detectado nuevas necesidades de cuantificación. La idea se repite en el Examen de Alto Nivel del Consejo de Seguridad ${ }^{48}$ en el que la representante de la Unión prometía una pronta renovación de los mismos. Así, en septiembre de 2016 se adoptaron 21 nuevos indicadores ${ }^{49}$, tras la publicación del Estudio Mundial, el informe del SG de 2015 y la Resolución 2242.

48 Actas del Examen de Alto Nivel «Las mujeres, la paz y la seguridad», 7533. a sesión, Consejo de Seguridad, martes 13 de octubre de 2015. S/Pv.7533, p. 61.

49 Revised indicators for the Comprehensive approach to the EU implementation of the UN Security Council Resolutions 1325 and 1820 on women, peace and security. 
La Women, Peace and Security Task Force identifica nuevos retos y prioridades, como el terrorismo y la violencia extrema (al hilo de los nuevos desafíos señalados por la Resolución 2242), la acción humanitaria en el contexto de desplazamientos de refugiados así como la necesidad de incrementar el presupuesto en la agenda MPS. Los 21 indicadores adoptados en 2016 se agrupan en diversas categorías, algunos se han dividido y otros se han clarificado, explicado o han aumentando su contenido a través de diferentes sub-indicadores ${ }^{50}$. La mejora de los indicadores es evidente por cuanto se refiere a su conexión con la realidad de las mujeres en las zonas de conflicto y posconflicto y a la implicación de todas las variables en cada uno de los objetivos. En el nuevo indicador número 2 se agrupan todas las categorías de personal relacionadas con la seguridad y defensa para analizar cuantitativamente el acceso a puestos de dirección así como destacar buenas prácticas, en su caso. También se otorga mayor importancia a la participación de las mujeres en los procesos de paz, a través de cuatro de ellos (del 11 al 14), así como a la Política Común de Seguridad y Defensa, otorgando más atención a los posibles casos de abuso y explotación (nuevo indicador número 18), a través de cinco sub-indicadores que inciden en la prevención, la reparación y la investigación.

Respecto a las mujeres refugiadas, mientras que en los antiguos indicadores sólo se requería un porcentaje por sexos de los solicitantes de asilo y la protección obtenida, en el nuevo indicador número 20 , se expresa la necesidad de proteger a las mujeres solicitantes de asilo, así como a promover buenas prácticas como medidas de protección y detección de desafíos, aunque se sigue sin querer sistematizar el análisis de la situación indagando en las causas por las cuales las mujeres solicitarían protección internacional. Se añade también a este nuevo cupo de indicadores una nueva preocupación, el número 21 , relativo a las políticas sensibles al género contra el extremismo violento, centrado en la prevención y el desarrollo de políticas sensibles al género adecuadas en los presupuestos de los Estados Miembros, así como la formación en género de los expertos de los EM en la lucha antiterrorista.

En cuanto a la inclusión de mujeres en los puestos de toma de decisiones en cuestiones de seguridad y defensa (uno de los objetivos principales del programa MPS que se refuerza en estos nuevos indicadores) ${ }^{51}$,

${ }^{50}$ Expert Policy Workshop: Refreshing Indicators for the EU's Women, Peace and Security Policy, EPLO, Bruselas, 2015.

${ }^{51}$ Guerrina, Roberta; Wright, Katharine A. M., «Gendering normative power Europe: lessons of the Women, Peace and Security agenda», International Affairs, 92 (2016): 293-312. 
hay que destacar que según el Estudio Mundial, en el año 2014 de los 28 puestos ejecutivos con que contaba el Servicio de Acción Exterior europeo (SEAE) sólo tres estaban ocupados por mujeres ${ }^{52}$, lo cual resulta bastante decepcionante dada la importancia de esta cuestión ${ }^{53}$. Actualmente, entre los nueve Enviados Especiales del SEAE no hay ninguna mujer, aunque se han creado nuevos puestos ejecutivos que hoy suman un total de 35 , estando 23 de ellos ocupados por mujeres ${ }^{54}$. Además, en octubre 2015 la Alta Representante de la UE crea la figura de Asesor Principal sobre cuestiones de género, recayendo el nombramiento sobre Mara Marinaki ${ }^{55}$ quien, en representación de la UE, asistió a los plenos sobre MPS en el Consejo de Seguridad en 2015 (con ocasión el Examen de Alto Nivel) y en 2016, en los que dio cuenta de los avances de la Organización. En la última sesión del Consejo de Seguridad sobre MPS, Marinaki enfatizaba los progresos realizados a través de los documentos e indicadores comentados, además de señalar la alianza estratégica que se había acordado con ONU Mujeres ${ }^{56}$ y anunciar que el primer informe de revisión de los nuevos indicadores se publicaría a finales del año 2016.

Igualmente cabe destacar como acciones de la Alta Representante, la inclusión de las consideraciones del programa MPS en la Estrategia Global para la política exterior y seguridad de la Unión Europea de 2016 (Estrategia Europea de Seguridad) ${ }^{57}$, aunque en la misma no se tome el género como una cuestión principal (objetivo) sí lo hace como una premisa transversal a través del acertado concepto de «enfoque pluridimensional», entre cuyas «dimensiones» se encuentra el género. En la Estrategia, además, se subraya la necesidad de fomentar «el papel de las mujeres en los esfuerzos de paz» ${ }^{58}$ y la participación de éstas en la elaboración de la política exterior ${ }^{59}$, afianzando también la imperativa relación entre el enfoque de género y los Derechos Humanos ${ }^{60}$, como se reafirma en el informe del Consejo sobre dicha Estrategia, «the protec-

52 Prevenir los conflictos..., op. cit., p. 258.

53 Where are the EU's Women Leaders in Foreign Affairs? One year on, EPLO (European Peacebuilding Liaison Office), 8 de marzo de 2016.

${ }^{54}$ Datos consultados en la página web del Servicio de Acción Exterior el 16 de noviembre de 2016 .

55 Actas del examen de alto nivel «Las mujeres, la paz y la seguridad», 7533. a sesión, Consejo de Seguridad, martes 13 de octubre de 2015. S/Pv.7533, p. 61.

56 Actas de la 7793. a sesión del Consejo de Seguridad de las Naciones Unidas, de 25 de octubre de 2016, S/PV.7793, p. 36.

57 Una visión común, una mirada conjunta: una Europa más fuerte. Estrategia Global para la política exterior y de seguridad en la Unión Europea, 2016.

58 Ibidem., p. 24.

59 Ibidem., p. 38.

60 Ibidem., p. 40. 
tion, fulfilment and the enjoyment of human rights by women and girls and the fight against gender-based violence remained high on the EU agenda» ${ }^{61}$.

En resumen, este marco común creado en la Unión Europea sobre el programa MPS requiere aún la asunción de compromisos concretos. La crisis de los refugiados en las fronteras de la Unión es un ejemplo de la falta de conexión entre esta cuantificación de género de (en este caso, de las solicitantes de asilo) y la falta de mecanismos de protección de las mujeres refugiadas, así como la falta de cautela en la aplicación del Derecho Internacional de los Refugiados y las recomendaciones del ACNUR sobre la persecución de género. De igual manera, la persistencia del techo de cristal y los errores en la implementación de la perspectiva de género en el interior de las misiones y operaciones pueden provocar la continuidad de un modelo masculinizado de la seguridad, que siga influyendo negativamente en la percepción de ésta por parte de las mujeres y niñas de las sociedades en conflicto y posconflicto. A todo ello hay que sumar la falta de información sobre los indicadores de muchos EM, lo que hace que los informes sobre éstos no puedan ofrecer una visión certera de la situación regional.

La actuación a nivel regional tiene una importancia creciente en el asunto MPS. Actualmente diversas Organizaciones regionales tienen un marco de actuación o plan de acción regional sobre MPS ${ }^{62}$. La Unión Europea se encuentra, junto a la Unión Africana y la OTAN, entre aquellas que han ofrecido un mayor desarrollo a los principales desafíos del mismo, dotándolos de un marco de referencia específico reflejo de las orientaciones de las Naciones Unidas. La Unión Africana adoptada su plan regional sobre MPS en el año $2009^{63}$ incorporando la transversalidad de género a todos los ámbitos de la Organización. La OTAN adoptada su primera política sobre MPS en 2007, y la directiva 40-1 en el año 2009, que pretendía integrar la dimensión de género a la organización militar de sus misiones ${ }^{64}$. Además, en el año 2010, adoptaba también un plan de acción.

La necesidad de que se integren las medidas en todos los niveles (internacional, regional e interno) responde al principio de coherencia y al de

${ }^{61}$ CFSP Report, Our Priorities in 2016, General Secretariat of the Council, 17 October 2016, 13026/16.

${ }^{62}$ La Comunidad Económica de los Estados de África Occidental, la Unión Europea, la Organización del Tratado del Atlántico Norte (OTAN), el Foro de las Islas del Pacífico, la Unión Africana, la Conferencia Internacional sobre la Región de los Grandes Lagos, la Organización para la Seguridad y la Cooperación en Europa (OSCE), la Liga de los Estados Árabes (LEA) y la Autoridad Intergubernamental para el Desarrollo.

63 African Union Gender Policy, Rev 2/Fev 10, 2009.

${ }^{64}$ Fletcher, F., «The Women, Peace and Security Agenda: Integrating a Gendered Perspective into Security Operation», World Affairs, vol. 41:1 (2017): 103. 
efectividad, sin ser ello óbice para que los Estados sigan llevando la mayor carga de responsabilidad en el desarrollo de estrategias y planes internos. Por ello, hay que poner atención en que las herramientas regionales puestas en marcha no queden sin su correspondiente refuerzo y asunción de responsabilidad por parte de los sus miembros. Es necesario que todos los Estados Miembros sigan el marco conceptual del programa MPS y refuercen los objetivos regionales con programas propios en sus estrategias de seguridad. Por ello, para completar este estudio a continuación será objeto especial de análisis el nuevo indicador número 1, que se refiere a los «Planes Nacionales Acción y otros documentos estratégicos en los EM», cuestión que ya se encontraba inserta en los indicadores de 2008 pero que ahora se dota de cuatro sub-indicadores, propuestos por la Women, Peace and Security Task Force: número de NAP (1.1), . $^{\circ}$ de documentos estratégicos (1.2), implicación de la sociedad civil en la elaboración e implementación (1.3) y propuesta de buenas prácticas (1.4).

\section{Planes Nacionales de Acción adoptados por los EM de la UE}

Una de las herramientas elementales para implementar las directrices MPS son los Planes Naciones de Acción (en adelante, PNA), en los que los Estados tienen la oportunidad de desarrollar las medidas recomendadas ${ }^{65}$ integrándolas en sus estrategias nacionales de seguridad. Así, los PNA eran objeto de análisis en la Unión Europea a través de los indicadores de 2008 (número 6) como ha lo han sido en los indicadores renovados de 2016, en los que aparece como número 1 y cuyo contenido va más allá de lo cuantitativo, como mencionamos arriba, a través de los nuevos sub-indicadores. La importancia de estas herramientas en los EM es central, ya que el compromiso adquirido con el programa MPS no puede desarrollarse sin una estrategia de género sistematizada en su agenda de seguridad y defensa.

A nivel global podemos identificar planes de tres tipos: de aquellos Estados que se encuentran en procesos de reconstrucción post-bélica o reconstrucción nacional, de aquellos Estados que, si bien son ajenos a estos procesos en su propio territorio, contribuyen a procesos de paz de terceros o, en tercer lugar, de aquellos Estados que no cooperan en procesos de paz de terceros pero deben añadir igualmente una dimensión de género en sus estrategias de seguridad. En el ámbito de la UE resulta de especial inte-

65 Vid., Gunrum, B. F:; Fritz, J. M., «Women, Peace and Security: An Analysis of the National Action Plans Developed in Response to UN Security Council Resolution 1325», en Societies Without Borders, Case Western Research University, vol. 4, issue 2 (2016): 209225 . 
rés detenerse a analizar el impacto de estos planes, que corresponderían en su mayoría al segundo grupo, comprobando si realmente éstos orientan su contribución a los escenarios de mantenimiento de la paz hacia el empoderamiento de las mujeres en zonas de conflicto y post-conflicto ${ }^{66}$.

Desde la aprobación de la Resolución 1325 en el año 2000 hasta la fecha ${ }^{67}, 63$ Estados han adoptado un PNA ${ }^{68}$, aunque muchos no lo han hecho hasta hace muy poco: Corea, Bosnia-Herzegovina, Gambia, Indonesia, Kosovo o Irak lo hacían en 2014 y Afganistán, Argentina, Palestina, Japón, Nueva Zelanda y Paraguay en 2015 y Kenia, Ucrania, Timor-Leste y Sudán de Sur en 2016. Además, otros Estados afirmaron su compromiso de adoptar los planes a lo largo de 2016, pese a que esto no ha sucedido: Angola, Argelia, Brasil, República Checa, Kazajistán, Letonia, Tailandia y Tanzania ${ }^{69}$. Europa y África son las regiones que se han mostrado mas sensibles a la elaboración y adopción de estos Planes, como evidencia el siguiente gráfico:

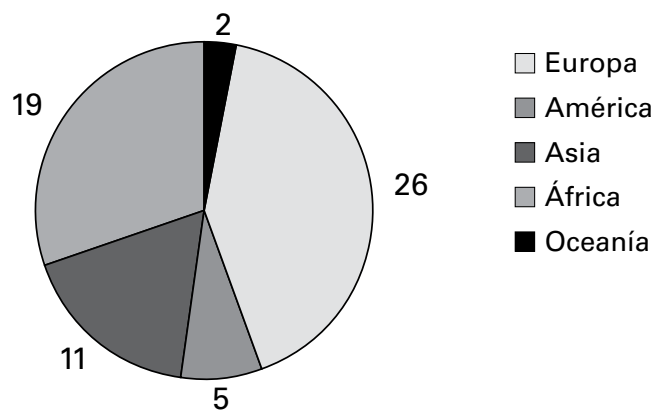

Planes nacionales de acción, por continentes

${ }^{66}$ SWAINE, A., «Assesing the Potential of National Action Plans to Advance Implementation of United Nations Security Council Resolution 1325», Yearbook of International Humanitarian Law, vol. 12 (2009): 403-433.

67 A diciembre de 2016.

${ }^{68}$ Son, por orden alfabético: Afganistán, Alemania, Argentina, Australia, Austria, Bélgica, Bosnia-Herzegovina, Burkina Faso, Burundi, Canadá, Chile, Corea, Costa de Marfil, Croacia, Dinamarca, Eslovenia, España, Estados Unidos, Estonia, Filipinas, Finlandia, Francia, Gambia, Gana, Georgia, Guinea, Guinea-Bissau, Holanda, Indonesia, Irak, Irlanda, Islandia, Italia, Japón, Kenia, Kosovo, Kirguistán, Liberia, Lituania, Macedonia, Malí, Nepal, Nigeria, Noruega, Nueva Zelanda, Palestina, Paraguay, Portugal, Reino Unido, República Centroafricana, República Democrática del Congo, Ruanda, Senegal, Serbia, Sierra Leona, Sudán del Sur, Suecia, Suiza, Takiyistán, Timor-Leste, Togo, Ucrania y Uganda.

${ }^{69} \mathrm{La}$ base de datos mundial sobre PNA está disponible en: http://www .peacewomen.org/ member-states. 
Vistos los datos por continentes destaca el liderazgo de la Europa y, en concreto, de la UE. Le sigue de cerca África, cuyos países han impulsado conjuntamente con Naciones Unidas numerosos instrumentos en este sentido. Es significativo que ambos contextos son aquellos cuyas organizaciones regionales han mostrado más interés hacia el programa MPS, contando con planes de acción regionales o documentos estratégicos, como comentamos anteriormente, el Planteamiento global de la Unión Europea y el African Union Gender Policy son muestras evidentes de la relación de sinergia positiva que suponen la acción regional hacia la estatal. En el polo opuesto, hay que destacar al continente americano, que tan sólo cuenta con los PNA de Canadá y Estados Unidos en el norte, y Chile, Argentina y Paraguay en el Sur. Respecto a Asia, se ha percibido un interés creciente que el Secretario General resaltó en su último informe, destacando el compromiso adquirido por los Estados en el simposio regional para la elaboración y revisión de Planes por parte de 17 Estados $^{70}$.

Los Estados miembros de la UE, los de la Asociación Europea de Libre Comercio (AELC) $)^{71}$ y los países europeos que no forman parte de la UE representan casi la mitad de los PNA del mundo: de los 26 PNA europeos, 17 son de Estados Miembros (EM) de la Unión, y 3 corresponden a Estados miembros de la AELC, excluyendo tan sólo a Liechtenstein, que no cuenta con un PNA. Respecto a los países de Europa que, por el momento, quedan fuera de la Unión Europea, existen 6 PNA adoptados: los de Macedonia, Serbia, Bosnia-Herzegovina, Kosovo, Georgia y Ucrania.

En todo caso, hay que tener en cuenta que la respuesta a este llamamiento entre los EM de la UE ha sido desigual: dieciséis años después de la adopción de la Resolución 1325 (2000), 11 EM no han adoptado hasta el momento un PNA, son: Bulgaria, Chipre, República Checa, Grecia, Hungría, Letonia, Malta, Polonia, Rumanía, Eslovaquia y Luxemburgo. No obstante, en los casos de Chipre y Malta éstos sí cuentan con documentos estratégicos ${ }^{72}$ para hacer valer las recomendaciones del programa «La mujer, la paz y seguridad ${ }^{73}$.

La adopción de dichos planes en el ámbito de la Unión es un factor clave para cumplir con las orientaciones comunes establecidas por el Con-

${ }^{70}$ Informe del Secretario General sobre las mujeres, la paz y la seguridad, S/2016/822, de 29 de septiembre de 2016, párr. 71.

71 Asociación Europea de Libre Comercio formada por Islandia, Noruega, Liechtenstein y Suiza.

72 UNSCR 1325 in Europe, 21 cases studies of implementation, EPLO, 2010.

73 Second Report on the EU-indicators for the Comprehensive Approach to the EU Implementation of the UN Security Council Resolutions 1325 \& 1820 on Women, Peace and Security, 30 de enero de 2014, p. 26. 
sejo. En este sentido, resulta muy relevante que 8 años después de la aprobación del Planteamiento global y tras ser un propósito incluido en los indicadores todavía existan 11 EM que no cuentan con un PNA, y que 9 de ellos no hayan aprobado ningún documento estratégico sobre el programa «la mujer, la paz y la seguridad». El último Estado Miembro que adoptó un PNA fue Alemania en el año 2013. De estos 11, 9 pertenecen también a la OTAN, en cuyo ámbito hay que sumar Albania y Turquía, quedando en un total de 11 Estados de la OTAN que no han cumplido con la recomendación de adoptar un PNA quince años después de la aprobación de la Resolución 1325 (de un total de 28). Hay que recordar además que, en los casos de Albania y Turquía, se trata de Estados candidatos a ingresar en la Unión Europea.

Volviendo a la UE, de los 17 EM que sí han aprobado un PNA, es interesante poner en valor las actualizaciones y nuevas adopciones que han tenido lugar en 12 de ellos, como podemos apreciar en la siguiente tabla:

\begin{tabular}{lccc}
\hline \multicolumn{1}{c}{ EM con PNA actualizado } & Primer PNA & Segundo PNA & Tercer PNA \\
\hline Austria & 2007 & 2012 & \\
Bélgica & 2009 & 2013 & \\
Dinamarca & 2005 & 2008 & 2014 \\
Estonia & 2010 & 2015 & \\
Finlandia & 2008 & 2012 & \\
Francia & 2010 & 2015 & \\
Holanda & 2008 & 2012 & \\
Irlanda & 2011 & 2015 & \\
Italia & 2010 & 2014 & \\
Portugal & 2009 & 2014 & \\
Reino Unido & 2006 & 2010 & 2014 \\
Suecia & 2006 & 2009 & 2016 \\
\hline
\end{tabular}

De estos 12 EM, en 9 casos ha habido una sola actualización del PNA, y en 4 casos (Dinamarca, Suecia, Reino Unido y Holanda) ha habido dos actualizaciones del mismo. En cuanto a los $5 \mathrm{EM}$ que no han actualizado o renovado su PNA, en la mayoría de los casos este hecho responde a razones temporales, ya que el mismo se ha adoptado más tarde y, en algunos casos, se encuentra actualmente vigente, aunque en otros ha expirado. No obstante, el único EM cuyo PNA se adoptó tempranamente y aún no ha sido renovado es España, como se puede apreciar en la siguiente tabla: 


\begin{tabular}{lc}
\hline \multicolumn{1}{c}{ EM que no han actualizado sus PNA } & Único PNA \\
\hline Alemania & 2013 \\
Croacia & 2011 \\
Eslovenia & 2010 \\
España & 2007 \\
Lituania & 2011 \\
\hline
\end{tabular}

Del informe sobre las mujeres, la paz y la seguridad del Secretario General de 2016 se desprende que ha habido una mejora en la interpretación de los PNA por parte de los Estados, así «en 45 de los planes (70\%) se han establecido mecanismos de seguimiento con indicadores de progreso y en $16(25 \%)$ se han fijado presupuestos de aplicación, lo que supone un ligero aumento respecto del $23 \%$ de $2014 \gg^{74}$. Valorándose positivamente que los Planes incluyan la denominada «rendición de cuentas por resultados» y no vagos e imprecisos propósitos sin metas cuantificables. Así, en los PNA más modernos que pueden apreciar una sistematización más integral de las acciones, así como la identificación de nuevos retos. Por ejemplo, en el PNA alemán es el único que incluye la persecución criminal como un área de preocupación prioritaria. Los Estados que han actualizado en plan tienden a corregir las deficiencias anteriores, como en el caso de Reino Unido, cuyo NAP de 2012 es el más específico hasta el momento, incluyendo sub planes para diferentes regiones (Afganistán, RDC y Nepal). Otro buen ejemplo es Holanda, quien ya en su segundo PNA (2012) incluía un presupuesto detallado (lo cual es muy poco común) y mejoraba la planificación y el monitoreo diseñados en el anterior ${ }^{75}$.

Una de las causas en la mejora de la interpretación de los planes puede encontrarse en la implicación cada vez mayor de los órganos de Derechos Humanos, que cada vez toman en mayor consideración el programa de MPS en sus recomendaciones a los Estados. Prueba de ello es que el 2015 nueve Estados recibieron recomendaciones por parte del Consejo de Derechos Humanos sobre sus políticas de MPS, incluyendo encomiendas de elaborar planes nacionales de acción ${ }^{76}$. La adopción de un PNA conlleva la

${ }^{74}$ Informe del Secretario General sobre las mujeres, la paz y la seguridad, S/2016/822, de 29 de septiembre de 2016, párr. 69.

75 Barbara Miller et. al., Women in Peace and Security through United Nations Security Resolution 1325: Literature Review, Content Analysis of National Action Plans and Implementation, IGIS WP 13/GGP WP 09 (Institute for Global and International Studies, May 2014): 122 .

76 Ibidem., párr. 74. 
asunción de compromisos primarios en la agenda de seguridad del Estado, pese a que éstos pueden variar desde una visión ampliamente militarista de la misma que se limite a fomentar la incorporación de mujeres en las fuerzas armados y otros, siguiendo las recomendaciones del Secretario General y del Estudio Mundial, otorguen prioridad a la formación sensible al género y la participación de asociaciones de mujeres en la elaboración del mismo, quedando el plan como un documento de consenso entre el Estado y la sociedad civil que siga las orientaciones de los ámbitos universal y regional. Sin duda, este último modelo de PNA es el más deseable, y sus expectativas seguramente se confirmarán con mayor asiduidad que las de aquellos Estados que han usado la elaboración de un PNA con objetivos más superficiales. Se analiza, finalmente, cuál es el modelo seguido por España.

\section{El Plan Nacional de Acción de España}

Respecto al desarrollo de las medidas propuestas en la Resolución 1325 y siguientes el gobierno español promulgó en noviembre de 2007 su Plan Nacional de Acción ${ }^{77}$. El caso de España es único en el ámbito de la UE, pues es el único ejemplo de un PNA aprobado con bastante rapidez pero no actualizado desde su aprobación en 2007. Su PNA era el quinto en adoptarse en el mundo (2007), y pese a que se aprobó un plan paralelo por parte de la AECID en 2009, el único a efectos oficiales es el adoptado en 2007.

En éste se incluían 6 puntos principales:

1. Fortalecer la participación de la mujer en las misiones de paz.

2. Promover la inclusión del enfoque de género en todas las actividades de la consolidación de la paz.

3. Asegurar la capacitación específica para el personal que participa en las operaciones de paz.

4. Proteger los derechos humanos de las mujeres y niñas en las zonas de conflicto y promover su participación en los procesos de paz.

5. Incorporar el principio de trato igualitario y oportunidades para las mujeres y los hombres en la planificación y la realización de actividades para el Desarme, la Desmovilización y la Reintegración.

6. Fomentar la participación de la sociedad civil española al respecto de la Resolución 1325.

77 Plan de Acción del Gobierno de España para la Aplicación de la Resolución 1325 del Consejo de Seguridad de las Naciones Unidas (2000), sobre Mujeres, Paz y Seguridad. http:// www.la-moncloa.es/ActualidadHome/301107-enlaceONU.htm. 
En efecto, en el año 2009 el Ministerio de Asuntos Exteriores y Cooperación aprobaba a través de la AECID un segundo plan para poner en marcha las disposiciones de la Resolución 1325 en el extranjero, a través de proyectos de cooperación al desarrollo. Se trata del Plan de Acción: Mujeres y Construcción de la Paz de la Cooperación Española ${ }^{78}$. En plan trata de proponer vías para el empoderamiento de las mujeres que viven en procesos continuos de construcción de la paz. Sin embargo, la carencia de recursos económicos asociados a este proyecto ha dejado en saco roto sus propósitos, lo que revela un posible uso cosmético ${ }^{79}$. Además, algunas asociaciones de mujeres de los países de destino han afirmado no haber sido consultadas con respecto a las políticas que el plan tendría preparadas para ellas, y sobre la influencia en sus vidas que éste tendría. Así pues, la falta de coherencia entre medidas a realizar y necesidades de las mujeres que viven en territorios dominados por el conflicto y la violencia no se han visto correspondidas con el pretendido enfoque de la «gobernanza» ${ }^{80}$.

El PNA español es, por su parte, bastante escueto y superficial, estando basado fundamentalmente en cuestiones de política exterior y no contando con sistemas de monitoreo y evaluación de objetivos ${ }^{81}$. No obstante, en los 3 primeros puntos del PNA se ha logrado un avance considerable con respecto a la situación anterior. Según el documento único del III y IV Informe de Seguimiento ${ }^{82}$, las mujeres constituyen actualmente un $12.5 \%$ de los efectivos de las Fuerzas Armadas, aunque no se ha llegado a eliminar el techo de cristal. Además, la LO 9/2011 de derechos y deberes de los miembros de las Fuerzas Armadas ${ }^{83}$ así como la Ley 39/2007 de la Carrera Militar ${ }^{84}$ han ofrecido importantes avances en cuanto al empoderamiento de las mujeres en Fuerzas Armadas, el entrenamiento en cuestiones sensibles al género y el establecimiento de incipien-

78 Se puede acceder al texto del plan en: http://www.aecid.es/galerias/programas/Vita/ descargas/PLANDEACCIONmujeresypaz.pdf.

79 Julia Espinosa Fajardo, «La igualdad de género en la cooperación española y en la iniciativa Marca España. Abordajes en inconsistencias en tiempos de crisis», Investigaciones Feministas, vol. 5 (2015): 259-288, 270.

${ }^{80}$ Picasso Mora, Ingrid, Gobernanza y Construcción de paz en la Cooperación Internacional para el Desarrollo de la Unión Europea en zonas en conflicto. El programa Nuevos Territorios de Paz en Colombia como caso de estudio (Instituto Mora, 2016).

81 Barbara Miller et. al., Women in Peace and Security..., op. cit., p.

82 Plan de acción del Gobierno de España para la aplicación de la Resolución 1325 del Consejo de Seguridad de las Naciones Unidas (2000) sobre Mujeres, paz y seguridad, III y IV Informe de Seguimiento, finalizado el 6 de febrero de 2014, pp. 133.

${ }^{83}$ LO 9/2011, de 27 de julio, de derechos y deberes de los miembros de las Fuerzas Armadas. «BOE» núm. 180 , de 28 de julio de 2011.

${ }^{84}$ Ley 39/2007, de 19 de noviembre, de la Carrera Militar. BOE núm. 278 de 20 de noviembre de 2007 
tes medidas de conciliación de la vida laboral y familiar. Incluso dando cuenta de estos avances es necesario destaca la impronta militarista del concepto de seguridad, que ha sido también puesta de manifiesto por diferentes organizaciones de la sociedad civil ${ }^{85}$.

Los puntos débiles de la PNA español son, sin duda, los relativos a incorporar el principio de trato igualitario y oportunidades para las mujeres y los hombres en la planificación y la realización de actividades para el Desarme, la Desmovilización y la Reintegración y a fomentar la participación de la sociedad civil española al respecto de la Resolución $1325^{86}$. Todo ello hace del PNA español un documento más que insuficiente.

Como una externalidad positiva de la elección de España como miembro no permanente del Consejo de Seguridad para el bienio 2015-2016, su interés en el programa MPS ha aumentado considerablemente en los últimos años. Como ya aludimos arriba, ha sido protagonista de iniciativas relevantes durante el Examen de Alto Nivel y ha otorgado un lugar primordial al programa en su agenda de seguridad. Como era evidente, había un asunto que debía atender urgentemente de querer postularse como Estado ejemplo de buenas prácticas sobre la perspectiva de género en la seguridad: la elaboración y adopción de un nuevo PNA, lo que ha sido ya anunciado, por lo que próximamente podremos comprobar si se han corregido los errores del primer PNA y se han asumido nuevos y más profundos compromisos ${ }^{87}$. Sin duda, en consonancia con las exigencias del Secretario General, el plan debe asumir responsabilidades cuantificables y rendición de cuentas por resultados, además de elaborarse en conjunto con asociaciones de mujeres y teniendo en cuenta las necesidades de las mujeres de los destinos en los que se van a desplegar las estrategias. Además, debe ser coherente con el marco regional y otorgar importancia a sus aportaciones sobre el programa MPS a la Política Común de Seguridad y Defensa.

\section{Conclusiones}

El programa «la mujer, la paz y la seguridad» se encuentra en una segunda fase de rendición de cuentas por resultados. Con un marco normativo

${ }^{85}$ María Villelas Ariño, Women, Peace and Security: 15 years of UN Security Council Resolution 1325. An Assement of the Women, Peace and Security Agenda, Reports 12/2016 (Institut Catalá Internacional per la Pau, 2016): 32, 17.

${ }_{86}$ Manuela Mesa, Las mujeres cuentan: informe de seguimiento sobre la aplicación de la Resolución 1325 en España, Documento de trabajo (CEIPAZ, Madrid, 2011).

87 Sobre lo que sería deseable que incluyera el segundo PNA de España Vid., María Solanas Cardín, «Ocho recomendaciones para el II Plan de Acción Nacional sobre Mujeres, Paz y Seguridad», ARI 69/2016, Real Instituto Elcano (30 de septiembre de 2016). 
comprehensivo de la mayor parte de desafíos, es necesario avanzar en los propósitos concretos en la línea del Grupo Oficioso sobre MPS del Consejo de Seguridad de las Naciones Unidas. La elaboración de indicadores es crucial para evidenciar la situación de discriminación existente en el ámbito de la paz y la seguridad a nivel universal y regional.

La acción regional de la UE ha sido amplia y coherente con las orientaciones de Naciones Unidas, permaneciendo a la cabeza del compromiso de las Organizaciones Internacionales regionales en este sentido. Sin embargo, los esfuerzos por aplicar las directrices del programa a las misiones y operaciones sobre el terreno son insuficientes, así como la gestión de crisis humanitarias que suceden en el propio territorio de la Unión. El techo de cristal sigue existiendo en los ámbitos militares y políticos de la PCSD, todo ello denota falta de congruencia en la protección de mujeres y niñas víctimas de conflictos armados, por ejemplo las refugiadas o las víctimas de trata.

Cuando se cumplen diecisiete años de la adopción de la Resolución 1325 (2000) podemos comprobar que el seguimiento estatal de las recomendaciones establecidas en ésta y sucesivas resoluciones sigue siendo insuficiente. No obstante, hay regiones que han liderado la aprobación de PNA: cabe destacar la importancia de los PNA aprobados en países africanos, puesto que, en su mayoría, se trata de territorios en fase de posconflicto. En cuanto a los Estados que participan en procesos de reconstrucción o mantenimiento de la paz en otros territorios, el papel de los EM de la Unión Europea es principal. La adopción de PNA en el ámbito europeo ha confirmado a esta región como líder en la adopción de medidas orientadas a aplicar las estrategias propuestas por el programa del Consejo de Seguridad «La mujer, la paz y la seguridad». Sin embargo, hay que subrayar que, pese a los instrumentos impulsados por el Consejo de la Unión Europea, aún existen $9 \mathrm{EM}$ que no cuentan con ningún tipo de plan o acción estratégica en este sentido, lo que supone casi un tercio de los EM de la Unión. Igualmente, el seguimiento de esta recomendación ha sido desigual en el ámbito de la OTAN, lo que resulta especialmente relevante por tratarse de una organización de fines específicamente orientados a la defensa y mantenimiento de la paz y seguridad.

En cuanto al PNA español, las notas negativas son su falta de actualización y, en ocasiones, falta de presupuesto en las acciones desplegadas concretamente por la AECID. No obstante, también hay luces que conviene destacar en cuanto al fortalecimiento del papel de las mujeres en las Fuerzas Armadas y la aplicación de un enfoque transversal de género en las actividades desplegadas en mantenimiento de la paz. España, comenzó teniendo un papel líder en la aplicación del programa, pero es necesario que actualice y dé coherencia a su estrategia sobre «La mujer, la paz y la seguridad». 


\section{Bibliografía}

Álvarez, Alejandro José, «Mujer, paz y seguridad y los nuevos desafíos a la seguridad global», VI Congreso de Relaciones Internacionales, La Plata (2016).

Barbé, Esther, «Supporting Practices Inspiring by Solidarist Ideas: The EU and the UNSC Open Debates on Women, Peace and Security», en EU Policy Responses to a Shifting Multilateral System, Barbé, Esther, et al. (Palgrave Macmillan, 2016): 135-156.

Espinosa Fajardo, Julia, «La igualdad de género en la cooperación española y en la iniciativa Marca España. Abordajes en inconsistencias en tiempos de crisis», Investigaciones Feministas, vol. 5 (2015): 259-288.

Fletcher, F., «The Women, Peace and Security Agenda: Integrating a Gendered Perspective into Security Operation», World Affairs, vol. 41, vol. 1 (2017).

Guerrina, Roberta; Wright, Katharine A. M., «Gendering normative power Europe: lessons of the Women, Peace and Security agenda», International Affairs, 92 (2016): 293-312.

Gunrum, B. F y Fritz, J. M., "Women, Peace and Security: An Analysis of the National Action Plans Developed in Response to UN Security Council Resolution 1325», en Societies Without Borders, Case Western Research University, vol. 4, issue 2 (2009): 209-225.

Kirby, Paul; Shepherd, Laura, «Reintroducing women, peace and security», International Affairs, 92 (2016): 249-254.

Mesa, Manuela, Las mujeres cuentan: informe de seguimiento sobre la aplicación de la Resolución 1325 en España, Documento de trabajo (CEIPAZ, Madrid, 2011).

Swaine, A., "Assesing the Potential of National Action Plans to Advance Implementation of United Nations Security Council Resolution 1325», Yearbook of International Humanitarian Law, vol. 12 (2009): 403-433.

Martín Martínez, Magdalena y Jiménez Sánchez, Carolina, «La protección internacional de los Derechos Humanos de las mujeres: una visión desde la multiculturalidad y la perspectiva de género», en Diversidad Cultural, Género y Derecho, ed. por Patricia Laurenzo Copello y Rafael Durán Muñoz (Tirant lo Blanch, 2014): 269-305.

Lombardo, Emanuela, «El mainstreaming de género en la Unión Europea», Aequalitas. Revista jurídica de igualdad de oportunidades entre mujeres $y$ hombres, vol. 10-15, mayo-diciembre (2003): 6-11.

Paffenholz et al., «Making Women Count: Assessing Women's Inclusion and Influence on the Quality and Sustainability of Peace Negotiations and Implementation», Geneva: Inclusive Peace and Transition Initiative (The Graduate Institute of International and Development Studies and UN Women, April 2016): 61.

Olsson, Louise et. al., Gender, Peace and Security in the European Union's Field Missions (Folke Bernardotte Academy, Stockholm, 2014): 65.

Amich Elías, Cristina, «Capacitación, monitoreo e indicadores», en Formación $y$ adiestramiento sensible al género del personal cívico-militar y el empoderamiento de la mujer, De Tomás Morales, Susana (Dir.) (Dyckinson y Ministerio de Asuntos Exteriores y Cooperación, 2015): 280. 
Jiménez Sánchez, Carolina, Las mujeres en los conflictos armados: conflicto, proceso de paz y posconflicto (SPYDUM, Málaga, 2016): 276.

Miller, Barbara et. al., Women in Peace and Security through United Nations Security REsolution 1325: Literature REview, Content Analysis of National Action Plans and Implementation, IGIS WP 13/GGP WP 09 (Institute for Global and International Studies, May 2014).

Solanas Cardín, María, «Mujeres, paz y seguridad: lejos de las aspiraciones de la Resolución 1325», ARI 44/2015, Real Instituto Elcano (15 de septiembre de 2015).

«Ocho recomendaciones para el II Plan de Acción Nacional sobre Mujeres, Paz y Seguridad», ARI 69/2016, Real Instituto Elcano (30 de septiembre de 2016).

Villelas Ariño, María, Women, Peace and Security: 15 years of UN Security Council Resolution 1325. An Assement of the Women, Peace and Security Agenda, Reports 12/2016 (Institut Catalá Internacional per la Pau, 2016): 32.

\section{Sobre el autor}

Carolina Jiménez Sánchez es Profesora Ayudante Doctora de Derecho Internacional Público y Relaciones Internacionales de la Universidad de Málaga y tutora de la UNED de Derecho Internacional y Derecho de la Unión Europea. Forma parte de varios proyectos de investigación sobre riesgos y amenazas a la seguridad global y sobre grupos marginados por el Derecho. En 2015 fue Premio International de Investigación Victoria Kent por una adaptación de su tesis, publicándose como monografía con el título «Las mujeres en los conflictos armados: conflicto, proceso de paz y posconflicto». Ha realizado diversas estancias internacionales de investigación en centros de excelencia entre los que se cuentan la Universidad de Cambridge (Reino Unido), el European University Institute (Florencia, Italia), el Transitional Justice Institute (Belfast, Reino Unido) o el Centre for Gender and Women's Studies (Lancaster, Reino Unido). Cuenta con diferentes publicaciones en español, inglés y francés en revistas y obras colectivas, así como con otras dos monografías, una sobre el conflicto del Sáhara Occidental (Eumed, 2014) y una tercera sobre la dimensión de género en los tribunales penales internacionales (Aranzadi, 2016). Sus principales líneas de investigación son la perspectiva de género en el Derecho Internacional, el refugio y el asilo y el conflicto del Sáhara Occidental.

\section{About the author}

Carolina Jimenez Sanchez is a lecturer on Public International Law and International Relations at the University of Malaga and Tutor Lecturer 
of National University of Distance Education. Public and Private International Law and European Law. She takes part in several research projects about new treats and risk on International Security as well as vulnerable group at the boundaries of Law. In 2013 she obtained her $\mathrm{PhD}$ with a thesis on gender and armed conflict. In 2015 won the International Research Award «Victoria Kent». She has made several international research stays at centers of excellence, among which the University of Cambridge, the Transitional Justice Institute, University of Ulster (Northern Ireland) or the European University Institute in Florence (Italy) or the Centre for Gender and Women's Studies (Lancaster, UK) and have published books and papers in the field of gender and International Law, Asylum and Refugee Law and Western Sahara conflict. She have two recently published books about women and war: «Las mujeres en los conflictos armados: conflicto, proceso de paz y posconflicto» (2015) and «La dimensión de género en los Tribunales Penales Internacionales» (2016). 


\section{Derechos de autor}

Los derechos de autor (para la distribución, comunicación pública, reproducción e inclusión en bases de datos de indexación y repositorios institucionales) de esta publicación (Cuadernos Europeos de Deusto, CED) pertenecen a la editorial Universidad de Deusto. El acceso al contenido digital de cualquier número de Cuadernos Europeos de Deusto es gratuito inmediatamente después de su publicación. Los trabajos podrán leerse, descargarse, copiar y difundir en cualquier medio sin fines comerciales y según lo previsto por la ley; sin la previa autorización de la Editorial (Universidad de Deusto) o el autor. Así mismo, los trabajos editados en CED pueden ser publicados con posterioridad en otros medios o revistas, siempre que el autor indique con claridad y en la primera nota a pie de página que el trabajo se publicó por primera vez en $C E D$, con indicación del número, año, páginas y DOI (si procede). Cualquier otro uso de su contenido en cualquier medio o formato, ahora conocido o desarrollado en el futuro, requiere el permiso previo por escrito del titular de los derechos de autor.

\section{Copyright}

Copyright (for distribution, public communication, reproduction and inclusion in indexation databases and institutional repositories) of this publication (Cuadernos Europeos de Deusto, CED) belongs to the publisher University of Deusto. Access to the digital content of any Issue of Cuadernos Europeos de Deusto is free upon its publication. The content can be read, downloaded, copied, and distributed freely in any medium only for non-commercial purposes and in accordance with any applicable copyright legislation, without prior permission from the copyright holder (University of Deusto) or the author. Thus, the content of $C E D$ can be subsequently published in other media or journals, as long as the author clearly indicates in the first footnote that the work was published in $C E D$ for the first time, indicating the Issue number, year, pages, and DOI (if applicable). Any other use of its content in any medium or format, now known or developed in the future, requires prior written permission of the copyright holder. 